\title{
Synchronisation of Canonical Measures for Hyperbolic Attractors
}

\author{
William Parry \\ Mathematics Institute, University of Warwick, Coventry CV4 7AL, England
}

\begin{abstract}
Under suitable conditions it is shown how to change the velocity of a $C^{2}$ Axiom $A$ attractor so that the Sinai-Ruelle-Bowen measure coincides with the measure of maximal entropy. These measures are obtained as limits of certain closed orbital measures.
\end{abstract}

There are two invariant (probability) measures which come to the fore in the study of $C^{2}$ hyperbolic attractors (and no doubt in the study of dynamical systems satisfying less stringent hyperbolic conditions). They are (i) the measure of maximal entropy and (ii) the Sinai-Ruelle-Bowen (S.R.B.) measure, which, in case the flow preserves a smooth measure is this measure. There are many examples where these two measures differ and many where they coincide.

The aim of this note is to show that it is possible, under suitable conditions, to change the velocity so that the corresponding measures for the new flow coincide. This change of velocity is brought about by the multiplication of speeds by a function which is essentially unique. In other words the "reason" for the two canonical measures differing appears to be that the system is running at the wrong speed and that there is a canonical speed (up to constant scalar factors) for the 1 dimensional foliation of the flow.

A secondary purpose of this note is to describe the S.R.B. measure from an internal point of view as opposed to the usual external definition in terms of the behaviour of (Lebesgue) almost all points. Our description is in terms of weighted orbital measures. Unweighted orbital measures converge weakly to the measure of maximal entropy according to a theorem of Bowen [4] whereas weighted orbital measures converge to the S.R.B. measure (for appropriate weights, of course). The latter fact has been observed and conjectured for certain systems by Hannay and Ozorio De Almeida [6].

In Sect. 5 we discuss, briefly, the Ruelle zeta function, which captures periodic orbital data and is an invariant of topological conjugacy. In contrast to this function we introduce (for $C^{2}$ hyperbolic attractors) a different zeta function which is invariant under $C^{1}$ velocity changes (but not topological conjugacy). Under appropriate conditions the synchronisation mentioned above yields a flow for 
which the two zeta functions coincide. The internal definition of the S.R.B. measure results from certain analytic properties of another zeta function.

We conclude with an account of the proof, due to Anosov and Sinai [3], that a smooth change in the velocity of an Anosov flow results in an Anosov flow. This is needed for our main result.

\section{Transforms of Invariants}

We begin by making some elementary observations of a topological dynamical nature.

Let $\varphi_{t}$ be a continuous flow on the compact metric space $\Omega$. If $l$ is a strictly positive continuous function defined on $\Omega$, we can define a new flow $\psi_{t}$ by multiplying speeds by $l$. The new flow has exactly the same oriented orbits as the old flow. To do this define $k(x)=l(x)^{-1}, k(x, t)=\int_{0}^{t} k\left(\varphi_{s} x\right) d s$, and let $l(x, t)$ be the inverse self-homeomorphism of $\mathbb{R}$, for each $x$, i.e. $t \equiv k(x, l(x, t)) \equiv l(x, k(x, t))$. Next one checks that $\psi_{t}(x) \equiv \varphi_{l(x, t)}(x)$ is a flow, which amounts to showing $\psi_{s}\left(\psi_{t}(x)\right)$ $\equiv \psi_{s+t}(x)$ or $\varphi_{l\left(\psi_{t} x, s\right)}\left(\varphi_{l(x, t)}(x)\right)=\varphi_{l(x, s+t)}(x)$ or $l\left(\psi_{t} x, s\right)+l(x, t)=l(x, s+t)$.

The latter condition can be expressed by saying that $l$ is a $\psi 1$-cocylce. Note also that $k$ is a $\varphi 1$-cocyle and $\varphi_{t}(x)=\psi_{k(x, t)}(x)$. Furthermore, $l$ can be continuously differentiated along flow lines and

$$
l(x, t)=\int_{0}^{t} l\left(\psi_{s} x\right) d s
$$

Ergodic Measures. If $\mu$ is an ergodic $\varphi$ invariant probability measure, then $\mu^{k}$ $\equiv k(x) \mu / \int k(x) d \mu$ is an ergodic $\psi$ invariant probability measure.

Cocycles. If $\alpha(x, t)$ is a $\varphi 1$-cocycle, then $\beta(x, t)=\alpha(x, l(x, t))$ is a $\psi 1$-cocycle. When $\alpha$ is a $\varphi$ coboundary (i.e. $\alpha(x, t)=\gamma\left(\varphi_{t} x\right)-\gamma(x)$ for some continuous $\gamma$ ) then $\beta(x, t)$ $=\gamma\left(\varphi_{l(x, t)} x\right)-\gamma(x)=\gamma\left(\psi_{t} x\right)-\gamma(x)$ so that $\beta$ is a $\psi$ coboundary.

Periodic Orbits. If $\tau$ is a $\varphi$ periodic orbit of $\varphi$ period $\lambda_{\varphi}(\tau)$, then $\tau$ is a $\psi$ periodic orbit of $\psi$ period $\lambda_{\psi}(\tau)=k\left(x, \lambda_{\varphi}(\tau)\right)$ for $x \in \tau$.

Equivalently $\lambda_{\varphi}(\tau)=l\left(x, \lambda_{\psi}(\tau)\right.$ for $x \in \tau$. The $\varphi$ invariant measure of total mass $\lambda_{\varphi}(\tau)$ concentrated on $\tau$ is denoted $m_{\tau}^{\varphi}$ and $m_{\tau}^{\psi}=k m_{\tau}^{\varphi}$.

Winding Numbers. Let $H^{1}(\Omega, \mathbb{Z})$ be the first cohomology group, interpreted as the Bruschlinsky group. Elements of $H^{1}(\Omega, \mathbb{Z})$ are continuous maps from $\Omega$ to $K$, the unit circle, modulo those which are homotopically constant. Elements of $H^{1}(\Omega, \mathbb{Z})$ can be represented by continuous maps which are continuously differentiable with respect to $\varphi$, where

$$
f_{\varphi}^{\prime}(x)=\lim _{t \rightarrow 0}\left(f\left(\varphi_{t} x\right)-f(x)\right) / t
$$

If $\mu$ is a finite $\varphi$ invariant Borel measure, define

$$
W_{\mu}^{\varphi}(f)=(1 / 2 \pi i) \int\left(f_{\varphi}^{\prime}(x) / f(x)\right) d \mu .
$$


This defines a homomorphism of $H^{1}\left(\Omega, \mathbb{Z}\right.$ ) into $\mathbb{R}$ (cf. [12]). The range of $W_{\mu}^{\varphi}$ is called the winding numbers group with respect to $\mu$. Since $f_{\psi}^{\prime}(x)=f_{\varphi}^{\prime}(x) . l(x)$, we have $W_{k \mu}^{\psi}=W_{\mu}^{\varphi}$. Notice that the range of $W_{\mu}^{\varphi}$ is a subgroup of $\mathbb{Z}$ when $\mu$ is the $\varphi$ invariant measure $m_{\tau}^{\varphi}$.

Entropy. Let $h(\varphi, \mu)=h\left(\varphi_{1}, \mu\right)$ denote the entropy of the flow $\varphi$ with respect to the ergodic $\varphi$ invariant probability measure $\mu$. Using a result of Abramov's (cf. [1]) one can show that

$$
h\left(\psi, \mu^{k}\right)=h(\varphi, \mu) / \int k d \mu .
$$

(Entropy is always defined with respect to probability measures.)

\section{Hyperbolic Attractors}

Let $\varphi_{t}$ be a $C^{1}$ flow defined on the compact Riemannian manifold $M$ and let $\Omega \subset M$ be a $\varphi$ invariant closed set with the following properties:

(i) $\varphi \mid \Omega$ is topologically transitive,

(ii) the periodic points of $\Omega$ are dense in $\Omega$ and there are no fixed points in $\Omega$,

(iii) there exists an open set $U \supset \Omega$ such that $\bigcap_{t \geqq 0} \varphi_{t} U=\Omega$,

(iv) $\varphi \mid \Omega$ is hyperbolic, i.e. $T_{\Omega} M=E^{u}+E^{s}+E^{0}$ (continuous splitting) where $\left\|D \varphi_{t} v\right\| \leqq C e^{-c t}\|v\|$, when $v \in E_{x}^{s}, t \geqq 0,\left\|D \varphi_{-t} v\right\| \leqq C e^{-c t}\|v\|$, when $v \in E_{x}^{u}, t \geqq 0, E_{x}^{0}$ is the one dimensional tangent space defined by the flow at $x$.

(v) $\Omega$ is non-trivial, i.e. it is not a topological circle.

Under these conditions $\varphi \mid \Omega$ is called a hyperbolic attractor. We shall impose stronger differentiability conditions as we need them. A flow which satisfies (iv) for $\Omega=M$ is called an Anosov flow. (In this definition the other conditions above are not assumed.)

When $\varphi$ is $C^{2}$ it is known that the hyperbolic splitting is Hölder continuous so that $D \varphi_{t} \mid E_{x}^{u}$ and $\lim _{t \rightarrow 0}(1 / t) \log \left|\operatorname{Jac}\left(D \varphi_{t} \mid E_{x}^{u}\right)\right|=\varphi^{u}(x)$ are Hölder continuous, where the Jacobian is defined with respect to the given Riemann metric (cf. [2, 5]). An improvement in the smoothness of $\varphi$ does not, in general, lead to an improvement in the smoothness of $\varphi^{u}$. If we assume that $\varphi$ is $C^{n}(n \geqq 2)$ and also assume that the hyperbolic splitting is $C^{n-1}$, then $\varphi^{u}(x)$ is $C^{n-1}$.

Proposition $1[13,5]$. When $\varphi$ is a $C^{2}$ hyperbolic attractor and $f$ is continuous, then for almost all $x \in U$ (with respect to the Riemann volume)

$$
\lim _{T \rightarrow \infty}(1 / T) \int_{0}^{T} f\left(\varphi_{t} x\right) d t=\int f d m_{\varphi}
$$

where $m_{\varphi}$ is the unique equilibrium state defined by $-\varphi^{u}$.

The measure $m_{\varphi}$ is called the Sinai-Ruelle-Bowen (S.R.B.) measure.

Corollary. If $\varphi$ is a $C^{2}$ hyperbolic attractor which preserves a probability measure $m$ which is absolutely continuous with respect to the Riemann volume on $U$ (i.e. $m$ is smooth on $U$ ) then $m=m_{\varphi}$. 
This is an immediate consequence of the proposition together with Birkhoff's ergodic theorem.

Remark. The measure $m_{\varphi}$ is independent of the Riemann metric chosen for $M$. This is deduced from the fact that a change of metric leads to a change in

$$
\varphi^{u}(x, t)=\int_{0}^{t} \varphi^{u}\left(\varphi_{s} x\right) d s=\log \left|\operatorname{Jac}\left(D \varphi_{t} \mid E_{x}^{u}\right)\right|
$$

by the addition of a coboundary.

Now let us change the velocity of $\varphi \mid \Omega$ by using a strictly positive $l \in C(\Omega)$ as in Sect. 1, to obtain the flow $\psi$ on $\Omega$. In general $\psi$ will no longer be a smooth flow, but it will be when $\Omega=M$ and $l$ is $C^{1}$. In any case we have

Corollary. If $\Omega=M$ (so that $\varphi$ is Anosov) and $f \in C(M)$, then for almost all $x \in M$

$$
\lim _{T \rightarrow \infty}(1 / T) \int_{0}^{T} f\left(\psi_{t} x\right) d t=\int f k d m_{\varphi} / \int k d m_{\varphi} .
$$

It is therefore reasonable to refer to the normalised measure $m_{\varphi}^{k}$ as the S.R.B. measure for $\psi$. A further reason is that when $l$ is $C^{2}, \psi^{u}$ is well defined and $m_{\psi}=m_{\varphi}^{k}$, as we shall see, from the fact that $\psi$ is a $C^{2}$ hyperbolic attractor.

Theorem 1 (Anosov and Sinai [3]). If $\varphi$ is a $C^{1}$ Anosov flow and $\psi$ is obtained from $\varphi$ by the multiplication of speeds by a positive $C^{1}$ function l, then $\psi$ is Anosov.

In addition we have

$$
\psi^{u}(x, s)=\varphi^{u}(x, l(x, s))+g\left(\psi_{s} x\right)-g(x)
$$

with g continuous.

The last statement is included for completeness. Anosov and Sinai (see Sect. 5) prove their result by constructing a hyperbolic splitting $T M=F^{u}+F^{s}+F^{0}$ for $D \psi_{s}$ as follows:

For a suitable real valued continuous function $z(x, v), v \in E_{x}^{u}$,

$$
F_{x}^{u}=\left\{v+z(x, v) X_{x}: X \text { is the vector field defining } \varphi\right\} .
$$

The function $z(x, v)$ is linear in the second variable. $F^{s}$ is defined in a similar way. Moreover

$$
D \psi_{s}\left(v+z(x, v) X_{x}\right)=D \varphi_{t} v+z\left(\varphi_{t} x, D \varphi_{t} v\right) X_{\varphi_{t}(x)},
$$

where $\psi_{s} x=\varphi_{t} x$, i.e. $t=l(x, s)$.

Now consider the maps of linear spaces

$$
E_{x}^{u} \rightarrow F_{x}^{u} \rightarrow F_{\psi_{s}(x)}^{u} \rightarrow E_{\varphi_{t}(x)}^{u}
$$

given by

$$
v \rightarrow v+z(x, v) X_{x} \stackrel{D \psi_{s}}{\longrightarrow} D \varphi_{t} v+z\left(\varphi_{t} x, D \varphi_{t} v\right) X_{\varphi_{t}(x)} \rightarrow D \varphi_{t} v
$$

with Jacobians $G(x), \operatorname{Det}\left(D \psi_{s} x\right), G\left(\varphi_{t} x\right)^{-1}$, so that

$$
\operatorname{Det}\left(D \varphi_{t} x\right)=G(x) \operatorname{Det}\left(D \psi_{s} x\right) G\left(\varphi_{t} x\right)^{-1}
$$


and with $g(x)=\log |G(x)|$ we have

$$
\varphi^{u}(x, t)=\psi^{u}(x, s)+g(x)-g\left(\varphi_{t} x\right) \quad \text { or } \quad \psi^{u}(x, s)=\varphi^{u}(x, l(x, s))+g\left(\psi_{s} x\right)-g(x) .
$$

An immediate consequence is that $\psi^{u}(\tau) \equiv \varphi^{u}(\tau)$, where $\varphi^{u}(\tau)=\varphi^{u}\left(x, \lambda_{\varphi}(\tau)\right), x \in \tau$. In other words

Proposition 2. Each $\varphi^{u}(\tau), \tau$ a closed orbit, is an invariant of velocity change by $C^{1}$ multiplication when $\phi$ is $C^{2}$.

\section{Equidistribution of Closed Orbits and Weighted Closed Orbits}

We shall need the following equidistribution theorems. The first one, which is due to Bowen [4], is unweighted, whereas the second one is weighted by $J_{\tau}^{u}=e^{\varphi^{u}(\tau)}$.

Proposition 3. If $\varphi$ is $C^{1}$ then the measure $\mu_{\varphi}$ of maximum entropy is the weak limit of the orbital measures $\Sigma_{\tau} m_{\tau}^{\varphi} / \Sigma_{\tau} \lambda_{\varphi}(\tau)$, where the summations are over closed orbits with periods $\lambda_{\varphi}(\tau)$ between $x$ and $x+\varepsilon$ and $x \rightarrow \infty$. (When $\varphi$ is not topologically weakmixing $\lambda_{\varphi}(\tau)$ is restricted to a closed discrete subgroup of $\mathbb{R}$.)

Theorem 2. When $\varphi$ is a $C^{2}$ attractor the S.R.B. measure $m_{\varphi}$ is the weak limit of the weighted orbital measures

$$
\Sigma_{\tau}\left(J_{\tau}^{u}\right)^{-1} m_{\tau}^{\varphi} / \Sigma_{\tau}\left(J_{\tau}^{u}\right)^{-1} \lambda_{\varphi}(\tau) .
$$

The proof of Theorem 2 is similar to the proof of Theorem 7 as presented in [9]. We shall comment further on the proof of this theorem in Sect. 5.

Remark. We have chosen to concentrate on two canonical measures - the equilibrium states of 0 and $-\varphi^{u}$, respectively. In fact, for every $t \in \mathbb{R}$ we might have considered the equilibrium state of $t \varphi^{u}$. The method sketched in Sect. 5 provides appropriately weighted orbital measures which converge weakly to this measure.

Problem. Is there a weighted version of Proposition 1 where the limiting integral is with respect to the measure of maximal entropy?

\section{Synchronisation}

Now let us suppose that $\varphi$ is a $C^{2}$ hyperbolic attractor with $\Omega=M$, and in addition that the sub-bundle $E^{u}$ is $C^{1}$. Then $\varphi^{u}$ is $C^{1}$ so that if we define $l(x)=K / \varphi^{u}(x)$, $k(x)=\varphi^{u}(x) / K(K$ a positive constant $)$ then $\psi$ is also $C^{1}$ and

$$
\begin{aligned}
& \psi^{u}(x, t)=\varphi^{u}\left(x, l(x, t)+g\left(\psi_{t} x\right)-g(x)=\right. K k(x, l(x, t))+g\left(\psi_{t} x\right)-g(x)=K t+g\left(\psi_{t} x\right)- \\
& g(x) .
\end{aligned}
$$

Hence $\psi^{u}(\tau)=\log J_{\tau}^{u}=K \lambda_{\psi}(\tau)$, from which we conclude, since

$$
\Sigma_{\tau}\left(J_{\tau}^{u}\right)^{-1} m_{\tau}^{\psi} /\left(\Sigma_{\tau}\left(J_{\tau}^{u}\right)^{-1} \lambda_{\psi}(\tau)\right) \sim \Sigma_{\tau} m_{\tau}^{\psi} /\left(\Sigma_{\tau} \lambda_{\psi}(\tau)\right),
$$

that

Theorem 3. Under the above conditions $m_{\psi}=\mu_{\psi}$. 
A more direct proof, perhaps, is given by noting that $-\psi^{u}$ and $-K$ have the same equilibrium states.

Moreover, there is only "one" $C^{1} \psi$ in the velocity class of $\varphi$ such that $m_{\psi}=\mu_{\psi}$.

By this we mean the following:

We know that $m_{\psi}=\mu_{\psi}$ when $\psi^{u}(x, t)=K t$ and then the topological entropy of $\psi$ is $K$. For this fixed $K$ if $\varrho$ has topological entropy $K$ and $m_{\varrho}=\mu_{\varrho}$ then the cocycle effecting a change from $\psi$ to $\varrho$ has the form $l(x, t)=t+g\left(\varrho_{t} x\right)-g(x)$. However, $\psi$ and $\varrho$ are topologically conjugate (as well as related by a velocity change) as is seen from the equation $\psi_{t}(\theta x)=\theta\left(\varrho_{t} x\right)$, when $\theta(x)=\psi_{-g(x)}(x)$.

Remark. The $C^{1}$ condition we have imposed on $E^{u}$ is a significant strengthening of the automatic Hölder condition associated with $C^{2}$ Anosov flows. As to its commonness the situation is not at all well understood. In [8] Hirsch, Pugh and Shub show that a $C^{2}$ Anosov flow such that $\operatorname{dim} E^{u}=1, \operatorname{dim} E^{s} \geqq 2$ has the property that $E^{u}$ is $C^{1}$, assuming that the flow preserves a smooth volume. Another class of examples where $E^{u}$ is $C^{1}$ is provided by the prototypical geodesic flows on surfaces of variable negative curvature. This is proved in [7] where it is also proved that if the curvature of a compact Riemannian manifold is negative and "absolutely 1/4-pinched" then the Anosov splitting for the geodesic flow is $C^{1}$. On the other hand Plante [11] has shown that small smooth perturbations of geodesic flows on surfaces of constant negative curvature generically (among those preserving a fixed volume) cannot have $C^{1}$ splittings. In a similar vein (and surprisingly in view of the construction of Anosov and Sinai, cf. Sect. 6) a generic smooth velocity change of a constant suspension of an Anosov diffeomorphism of the 2 torus, cannot have both sub-bundles $E^{u}, E^{s}$ satisfying the $C^{1}$ condition. There seems to be at least some possibility that the difficulty in achieving our condition is peculiar to low dimensional flows.

A further point to note is that we have imposed the $C^{1}$ condition on $E^{u}$ in order that the velocity changed flow (after synchronisation) remains a $C^{1}$ Anosov flow. Had we relinquished this requirement we would have been forced to consider Hölder flows. The essential content of this paper would remain intact had we decided to allow such flows into our considerations.

Let us assume that $\varphi$ is $C^{2}$ so that $\varphi^{u}$ is Hölder, and let $l(x)=K / \varphi^{u}(x)$, $k(x)=\varphi^{u}(x) / K$, where $K$ is a positive constant. Then $\psi_{t}(x)=\varphi_{l(x, t)}(x)$ is a well defined Hölder flow with the same oriented orbits as $\varphi$. The S.R.B. measure $m_{\psi}=m_{\varphi}^{k}$ is well defined and it is easy to see that $m_{\psi}=\mu_{\psi}$, the measure of maximal entropy for $\psi$. Indeed, by Abramov's formula we have to show that

$$
h\left(\psi, m_{\varphi}^{k}\right) \equiv h\left(\varphi, m_{\varphi}\right) / \int k d m_{\varphi} \geqq h\left(\psi, p_{\varphi}^{k}\right)=h\left(\varphi, p_{\varphi}\right) / \int k d p_{\varphi}
$$

for all ergodic $\varphi$ invariant probabilities $p_{\varphi}$, or equivalently that

$$
\left(h\left(\varphi, m_{\varphi}\right)-\int \varphi^{u} d m_{\varphi}\right) / \int \varphi^{u} d m_{\varphi} \geqq\left(h\left(\varphi, p_{\varphi}\right)-\int \varphi^{u} d p_{\varphi}\right) / \int \varphi^{u} d p_{\varphi} .
$$

However, the (numerator of the) left-hand side of this inequality is zero since it represents the pressure of $-\varphi^{u}$ and $m_{\varphi}$ is the equilibrium state for $-\varphi^{u}$. Hence the inequality follows from the variational principle.

I am grateful to Mark Pollicott for discussions concerning these problems and to the referee for asking for an elaboration of our $C^{1}$ condition. 


\section{Zeta Functions}

The Ruelle zeta function of $\varphi$ is defined as

$$
\zeta_{\varphi}(s)=\Pi_{\tau}\left(1-e^{-h \lambda_{\varphi}(\tau) s}\right)^{-1},
$$

where the product is over all closed orbits $\tau$ and where $h$ is the topological entropy of $\varphi \mid \Omega$. For $\varphi$ restricted to a basic set $\Omega$ of an Axiom $A$ flow (in particular for $\varphi$ restricted to a hyperbolic attractor) $\zeta_{\varphi}$ is non-zero and analytic for $\mathfrak{R}(s)>1$. When $\varphi \mid \Omega$ is topologically weak-mixing $\zeta_{\varphi}$ has a non-zero analytic extension to $\mathfrak{R}(s)=1$ except for a simple pole at $s=1$ (cf. [10]). It is clear that $\zeta_{\varphi}$ is an invariant of topological conjugacy but it is not invariant under velocity changes.

Here we introduce, what might be called a differential zeta function. If $\varphi$ is a $C^{2}$ hyperbolic attractor with $\Omega=M$ we define

$$
\zeta_{\varphi}^{u}(s)=\Pi_{\tau}\left(1-\left(J_{\tau}^{u}\right)^{-s}\right)^{-1}
$$

It can be shown, in much the same way as for the corresponding result for $\zeta_{\varphi}$, that $\zeta_{\varphi}^{u}$ has a non-zero analytic extension to $\mathfrak{R}(s) \geqq 1$ except for a simple pole at $s=1$, (when the changed flow $\psi$, discussed in Sect. 4, is topologically weak-mixing i.e., when $\left\{\varphi^{u}\left(x, \lambda_{\varphi}(\tau): \tau\right.\right.$ is a closed orbit $\}$ does not consist of integral multiples of a fixed number.) Since $J_{\tau}^{u}$ is invariant under $C^{1}$ changes in velocity, it is clear that $\zeta_{\varphi}^{u}$ is an invariant of such velocity changes. However, $\zeta_{\varphi}^{u}$ is not an invariant of topological conjugacy - unless the conjugacy is smooth.

When the flow $\varphi$ is synchronised, so that $\varphi^{u}(x, t)=h t+g\left(\varphi_{t} x\right)-g(x)$, the zeta functions $\zeta_{\varphi}, \zeta_{\varphi}^{u}$ coincide. This is the case, for example, with a geodesic flow on a surface of constant negative curvature. One wonders whether synchronisation has something to do with the very desirable features of the zeta function of such a geodesic flow?

The proof of Theorem 2 is based on another zeta function.

Let $\varphi \mid \Omega$ be a $C^{2}$ topologically weak-mixing attractor and let $f$ be a $C^{1}$ function defined on $M$.

Define (for each $\varepsilon>0$ )

$$
\zeta(s, z)=\prod_{\tau}\left(1-\exp \left(-\varphi^{u}(\tau)+z \lambda_{f}(\tau)+\varepsilon(1-s) \lambda_{\varphi}(\tau)\right)\right)^{-1}
$$

where $\lambda_{f}(\tau)=\int_{\tau} f\left(\varphi_{t} x\right) d t, x \in \tau$.

The proof of the following theorem is similar to the proof of Theorem 3 in [9].

Theorem 4. For each $s_{0} \in \mathbb{C}$ with $\mathfrak{R}\left(s_{0}\right) \geqq 1, s_{0} \neq 1, \zeta(s, z)$ is non-zero and analytic (or has such an extension) in a neighbourhood of $\left(s_{0}, 0\right)$. There is an analytic function $Q(s, z)$ such that

(i) $\zeta(s, z)\left(1-e^{Q(s, z)}\right)$ is non-zero and analytic in a neighbourhood of $(1,0)$,

(ii) $Q(1,0)=0, Q_{1}(1,0)=\int f d m_{\varphi}, Q_{2}(1,0)=\varepsilon$.

By taking logarithmic derivatives we obtain

$$
\eta(s) \equiv \zeta_{2}(s, 0) / \zeta(s, 0)=\Sigma_{\tau} \sum_{n=1}^{\infty} \lambda_{f}(\tau) \exp -n\left(\varphi^{u}(\tau)+\varepsilon(s-1) \lambda_{\varphi}(\tau)\right) .
$$


Again, proceeding as in [9], we obtain

\section{Theorem 5.}

$$
\Sigma_{\lambda_{\varphi}(\tau) \leqq x} \lambda_{f}(\tau) \exp \left(-\varphi^{u}(\tau)+\varepsilon \lambda_{\varphi}(\tau)\right) \sim \int f d m_{\varphi}\left(e^{\varepsilon x} / \varepsilon\right)
$$

as $x \rightarrow \infty$.

An immediate consequence is that

$$
\sum_{x<\lambda_{\varphi}(\tau) \leqq x+\delta} \lambda_{f}(\tau)\left(J_{\tau}^{u}\right)^{-1} e^{\varepsilon \lambda_{\varphi}(\tau)} \mid \sum_{x<\lambda_{\varphi}(\tau) \leqq x+\delta} \lambda_{\varphi}(\tau)\left(J_{\tau}^{u}\right)^{-1} e^{\varepsilon \lambda_{\varphi}(\tau)}
$$

converges weakly to $\int f d m_{\varphi}$ for every $\varepsilon>0$. The same is therefore true with $\varepsilon=0$, and hence Theorem 2 follows.

\section{The Anosov-Sinai Theorem}

We take the liberty of presenting a slightly modified account of the proof, due to Anosov and Sinai of Theorem 1.

Following the notation already established we suppose that $\varphi$ is a $C^{1}$ Anosov flow, that $l$ is a strictly positive $C^{1}$ function and that $k(x)=l(x)^{-1}$.

The flow obtained from $\varphi$ by multiplying speeds by $l$ is denoted $\psi$ and is also a $C^{1}$ flow.

For a fixed $x$ let $t=l(x, s)$ and $s=k(x, t)$ so that $\psi_{s}(x)=\varphi_{t}(x)$. We find a function $z(x, \xi)$ defined on the tangent bundle of $M$ such that $z(x, \cdot)$ is linear and

$$
D \psi_{s}\left(\xi+z(x, \xi) X_{x}\right)=D \varphi_{t} \xi+z\left(\varphi_{t} x, D \varphi_{t} \xi\right) X_{\varphi p: x},
$$

when $\xi$ is a tangent vector at $x$ with $\xi \in E_{x}^{\text {s. }}$. To find such a function we apply the lefthand side derivation to a smooth function $f$ to obtain $\left(\xi+z(x, \xi) X_{x}\right)\left(f \circ \psi_{s}\right)$. When $s=0$ we obtain $\left(\xi+z(x, \xi) X_{x}\right) f$ and the difference is

$$
\left(\xi+z(x, \xi) X_{x}\right)\left(f \circ \psi_{s}-f\right) .
$$

If we consider the right-hand side of (6.1) instead, we obtain

$$
\xi\left(f \circ \varphi_{t}-f\right)+z\left(\varphi_{t} x, D \varphi_{t} \xi\right) X_{\varphi_{t} x}(f)-z(x, \xi) X_{x}(f) .
$$

Dividing the supposed equal quantities (6.2), (6.3) by $s=k(x, t)$ and letting $s, t$ $\rightarrow 0$ we have

$$
(\xi+z X)(l X(f))=l\left(\xi X(f)+z^{\prime} X(f)+z X^{2}(f)\right),
$$

where $z^{\prime}$ denotes differentiation with respect to the flow $(x, \xi) \rightarrow\left(\varphi_{t} x, d \varphi_{t} \xi\right)$. Hence

$$
(\xi+z X)(l(X f))+l(\xi+z X)(X f)=l z^{\prime} X(f)+l(\xi+z X)(X f),
$$

i.e. $(\xi+z X)(l)=l z^{\prime}$.

Thus $z^{\prime}=z \cdot k \cdot X(l)+k \xi(l)$.

To solve this equation, and find the required function $z$, we consider the homogeneous version $W^{\prime}(x, \xi)=W(x, \xi) k(x) X_{x}(l)$. Clearly $l\left(\varphi_{t} x\right)$ is a solution, which suggests, by "variation of constants", that

$$
z\left(\varphi_{t} x, D \varphi_{t} \xi\right)=l\left(\varphi_{t} x\right) \cdot C(t)
$$


is an appropriate solution of (6.4) for some function $C(t)$. We consider, therefore,

$$
z^{\prime}=l^{\prime} \cdot C+C^{\prime} \cdot l=l k X(l) C+C^{\prime} \cdot l=z k X(l)+C^{\prime} \cdot l=z k X(l)+k \xi(l) .
$$

The function $C(t)$ should therefore satisfy

$$
C^{\prime}(t)=\xi(l) / l^{2} \text {, i.e. } C(t)=\int_{0}^{t}\left(D \varphi_{u} \xi\right) l /\left(l \circ \varphi_{u}\right)^{2} d u+C(0),
$$

which depends linearly on $\xi$. By requiring that $C(\infty)=0$, we have

$$
\left.z(x, \xi)=-l(x) \int_{0}^{\infty}\left(D \varphi_{u} \xi\right)(l) / l\left(\varphi_{u} x\right)^{2}\right) d u=l(x) \int_{0}^{\infty} \xi\left(k \circ \varphi_{u}\right) d u,
$$

which converges since $\xi \in E_{x}^{s}$. The function $z$ is clearly continuous and we define $F_{x}^{s}=\left\{\xi+z(x, \xi) X_{x}: \xi \in E_{x}^{s}\right\}$. By (6.1) we have $D \psi_{s} F_{x}^{s}=F_{\psi_{s}(x)}^{s}$, i.e. $D \psi_{s} F^{s}=F^{s}$.

The sub-bundle $F^{u}$ is defined in a similar way and $F^{0} \stackrel{=}{=} E^{0}$, by definition. It is not difficult to check that

$$
T M=F^{u}+F^{s}+F^{0}
$$

is a hyperbolic splitting for $\psi$, so that Theorem 1 is established.

\section{References}

1. Abramov, L.M.: On the entropy of a flow. Am. Math. Soc. Transl. 49, 167-170 (1966)

2. Anosov, D.V.: Geodesic flows on closed Riemann manifolds with negative curvature. Proceedings of the Steklov Institute 90 (1967)

3. Anosov, D.V., Sinai, Ya.G.: Some smooth ergodic systems. Russ. Math. Surv. 22, 103-167 (1967)

4. Bowen, R.: The equidistribution of closed geodesics. Am. J. Math. 94, 413-423 (1972)

5. Bowen, R., Ruelle, D.: The ergodic theory of axiom $A$ flows. Invent. Math. 29, 181-202 (1975)

6. Hannay, J.H., Ozorio DeAlmeida, A.M.: Periodic orbits and a correlation function for the semiclassical density of states. J. Phys. A: Math. Gen. 17, 3429-3440 (1984)

7. Hirsch, M., Pugh, C.: Smoothness of horocycle foliations. J. Differ. Geom. 10, 225-238 (1975)

8. Hirsch, M., Pugh, C., Shub, M.: Invariant manifolds. Lecture Notes in Math., Vol. 583. Berlin, Heidelberg, New York: Springer 1976

9. Parry, W.: Bowen's equidistribution theory and the Dirichlet density theorem. Ergodic Theory Dyn. Syst. 4, 117-134 (1984)

10. Parry, W., Pollicott, M.: An analogue of the prime number theorem for closed orbits of axiom $A$ flows. Ann. Math. 118, 573-591 (1983)

11. Plante, J.F.: Anosov flows. Am. J. Math. 94, 729-754 (1972)

12. Schwartzmann, S.: Asymptotic cycles. Ann. Math. 66, 270-284 (1957)

13. Sinai, Ya.G.: Gibbs measures in ergodic theory. Russ. Math. Surv. 166, 21-690 (1972)

Communicated by J. P. Eckmann

Received November 7, 1985; in revised form April 4, 1986 
